\title{
Transferring iodine: more than a simple functional group exchange in organic synthesis*
}

\author{
José Barluenga \\ Instituto Universitario de Química Organometálica 'Enrique Moles' - Unidad Asociada al \\ CSIC, Universidad de Oviedo, E-33071, Oviedo, Spain
}

\begin{abstract}
Some synthetic features of the $\mathrm{IPy}_{2} \mathrm{BF}_{4}$ reagent are presented. Among others, its utility to promote unusual 'carbon-carbon' coupling processes will be discussed. Furthermore, the unique iodinating ability of this reagent towards aromatic and other unsaturated systems will be summarized.
\end{abstract}

The rich chemistry of the carbon-iodine bond has made it a particularly rewarding synthetic tool of routine use for the purpose of functional group interconversion. Iodinated compounds are also credited as helpful diagnostic aids when the radioactive isotopes of the element are used [1]. Moreover, the growing presence and impact of hypervalent iodine compounds represents an additional source of interest in iodine containing reagents [2].

The bis(pyridinium) iodonium (I) tetrafluoroborate $\left(\mathrm{IPy}_{2} \mathrm{BF}_{4}\right)$ is a stable and solid reagent, that acts as a mild source of iodonium ions towards different types of unsaturated compounds, as it will be shown in this article. Initial efforts were devoted to the development of a methodology that can be useful to accomplish vicinal iodofunctionalization of an alkene. Thus, when the reagent is mixed in $\mathrm{CH}_{2} \mathrm{Cl}_{2}$ with an alkene and a nucleophile the corresponding products derived from the addition of an iodine atom and the nucleophile across the double bond are formed, for instance $\mathrm{MeOH}$ for 1a, in Scheme 1 [3]. Usually, an acid is required to neutralize the supply of pyridine molecules from the iodinating reagent, avoiding the formation of adducts, such as $\mathbf{1 b}$, that incorporate pyridine as a nucleophile through a competition process for the capture of the intermediate iodonium ion. In this regard, tetrafluoroboric acid is very useful due to the low nucleophilic character of the $\mathrm{BF}_{4}^{-}$counteranion. When simply the reagent, the acid and the alkene are mixed at low temperature, a clean, regio- and stereoselective iodofluorination takes place [3,4], as also depicted in Scheme 1 for the case of $\mathbf{1 c}$.
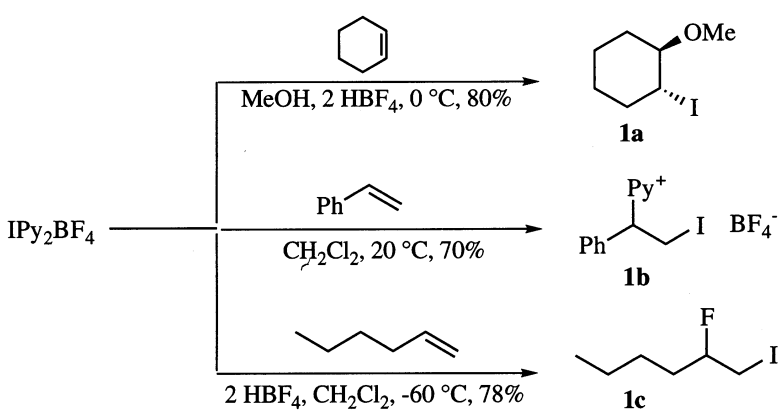

Scheme 1

\footnotetext{
*Lecture presented at the 5th International Conference on Heteroatom Chemistry (ICHAC-5), London, Ontario, Canada, 5-10 July 1998, pp. 369-512.
} 
Addition is the usual outcome of the reaction of $\mathrm{IPy}_{2} \mathrm{BF}_{4}$ with alkenes. However, starting from vinylsilanes an easy substitution process occurs, resulting in a very efficient and simple procedure for the iodo-silicon exchange from $E \mathbf{2 a}$, and $Z \mathbf{2 b}$ monosubstituted trimethylsilylalkenes, giving rise stereospecifically to the formation of compounds $\mathbf{3 a}$ and $\mathbf{3 b}$, respectively (Scheme 2) [5]. Otherwise, this is known to be an elusive transformation.

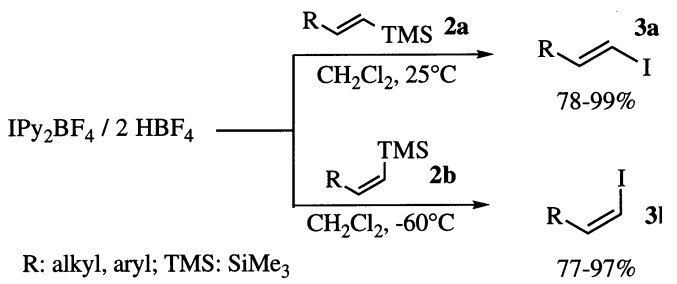

\section{Scheme 2}

Interestingly, the reagent is also an efficient iodinating agent promoting addition reactions of alcohols to the same alkenylsilanes furnishing compounds 4 (Scheme 3), by simply running the transformation in the presence of the corresponding alcohol as cosolvent [6]. The resulting adducts can be dehydroiodinated with the tertiary base 1,8-diazobicyclo[5.4.0]undec-7-ene (DBU), giving the stereochemically well-defined trisubstituted enol ethers, in a stereospecific manner.

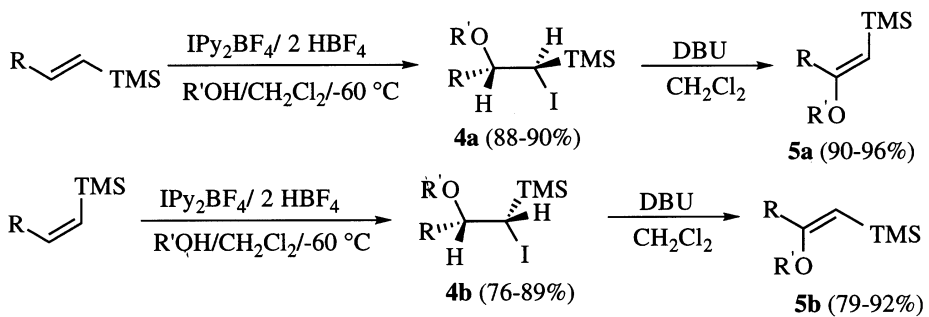

R: alkyl; R': Me, ${ }^{\mathrm{i}} \mathrm{Pr},{ }^{\mathrm{t}} \mathrm{Bu}$

\section{Scheme 3}

The same sequence, but requiring $t$-butyldimethylsilyl derivatives, gives aryl-substituted enol ethers. Adducts $\mathbf{6 a}$ and $\mathbf{6 b}$ are stereospecifically formed [6], now by a syn addition (Scheme 4).
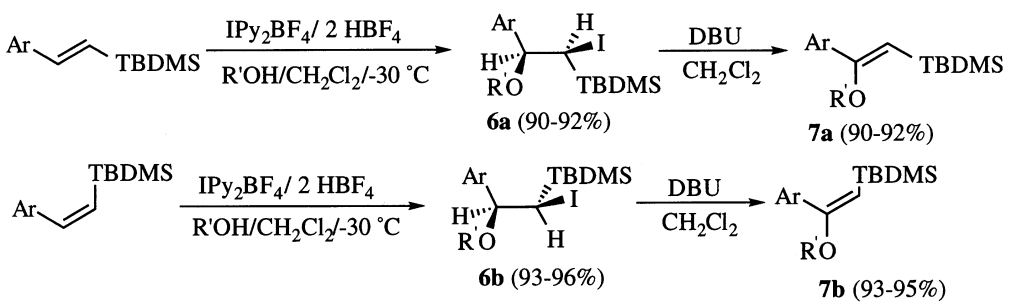

Ar: aryl; R': Me, ${ }^{\mathrm{P}} \mathrm{Pr},{ }^{t} \mathrm{Bu}$; TBDMS: $\mathrm{SiMe}_{2} \mathrm{Bu}^{\mathrm{t}}$

\section{Scheme 4}

It was reported that the $\mathrm{IPy}_{2} \mathrm{BF}_{4}$ reagent promotes electrophilic addition of iodine and nucleophiles to alkynes in a similar way, a process where the stereochemistry of the resulting alkenyliodides relies on the structure of both the alkyne and the nucleophile [7]. These functionalization reactions can be equally achieved using heteroatom-substituted alkynes as iodonium acceptors. The regiochemistry of the products formed upon addition to these substrates nicely illustrates the electronic tuning of the process (Fig. 1) [8]. Thus, 1-iodoalkynes are transformed into 2-functionalized-1,1-diiodoalkenes, whereas for alkynylsulfides the regiochemistry switches, yielding the corresponding 2-iodovinylsulfides [8b]. 


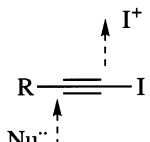

$\mathrm{Nu} * i$

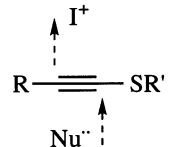

$\mathrm{Nu} \ddot{i}$

Fig. 1

A remarkable feature of the reagent relates to its usefulness mediating carbon-carbon coupling reactions. It was shown that, at low temperature, $\mathrm{IPy}_{2} \mathrm{BF}_{4}$ promotes novel types of Friedel-Crafts-like coupling reactions between alkenes or alkynes and arenes or another unsaturation [9,10], as outlined in Scheme 5 for the conversion of compounds 8-10 into 15, 16 (requiring trifluoromethane sulfonic, rather than tetrafluoroboric acid) and $\mathbf{1 7}$, respectively.

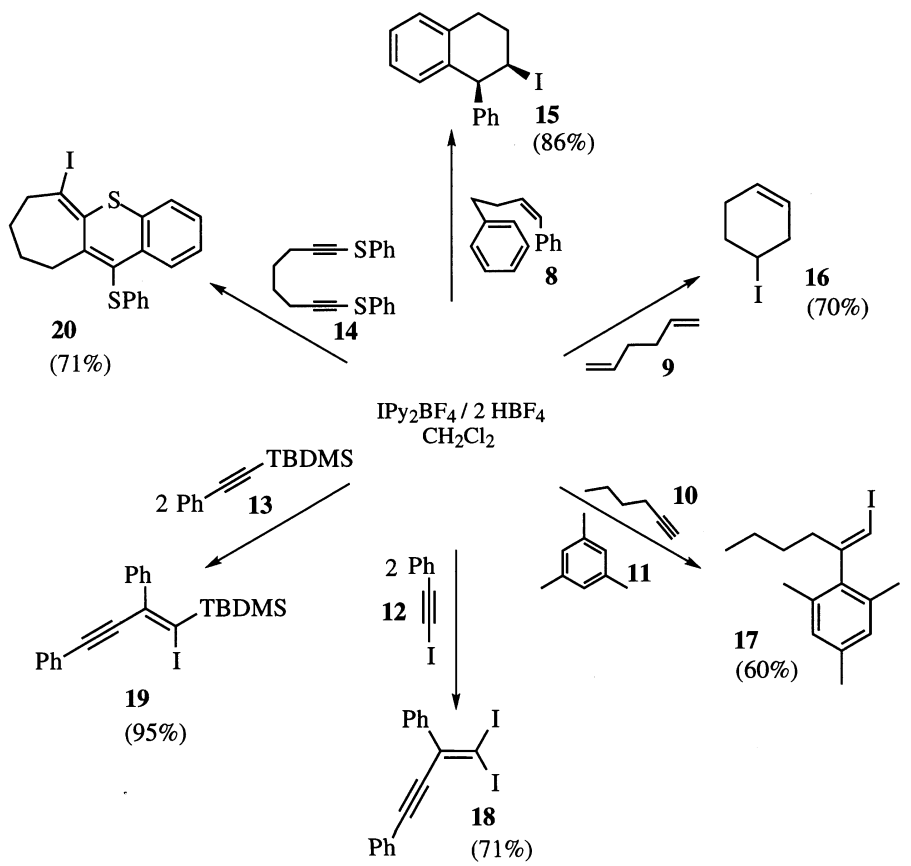

\section{Scheme 5}

Further studies directed to explore the potential of the reagent for the coupling of alkynes are also outlined in Scheme 5. The conversion of iodoalkyne $\mathbf{1 2}$ into the dimer $\mathbf{1 8}$ is an example of the potential of $\mathrm{IPy}_{2} \mathrm{BF}_{4}$ to selectively promote a homocoupling reaction, corresponding in this case to a head-to-tail mode, from this type of heteroatom-substituted alkyne [11]. This process, though limited to aryl and to alkenyl substituted alkynes, is relevant because transition metal complexes, the standard catalyst for coupling reactions of alkynes, do not offer an alternative to accomplish this transformation from that starting material. Besides, as the dimerization of 2-aryl-1-iodoalkynes is catalytic in the iodonium source, it represents another example of 'atom economy' transformations [12].

Modifying the heteroatom substituent on the starting alkyne, moving from iodine to silicon (as a tertbutyldimethylsilyl group), and optimizing conversion time and temperature, the reaction with $\mathrm{IPy}_{2} \mathrm{BF}_{4}$ leads almost quantitatively to a different head-to-tail dimer, as depicted in Scheme 5 for the conversion 13-19 (now the molar ratio alkyne: $\mathrm{IPy}_{2} \mathrm{BF}_{4}: \mathrm{HBF}_{4}$ is $1: 1: 1$ ) [13]. The enyne, contains two different heteroatoms at $\mathrm{C}-1$, and is formed with control over both, regio-and stereochemistry. Moreover, at higher temperatures, $\mathbf{1 9}$ or the enyne $\mathbf{2 1}$ (prepared from a modification of the above described dimerization, yielding the cross-coupling product from $4-\mathrm{Cl}-\mathrm{C}_{6} \mathrm{H}_{4} \mathrm{C} \equiv \mathrm{CTBDMS}$ and $\mathrm{PhC} \equiv \mathrm{CI}$ ) further react with $\mathrm{IPy}_{2} \mathrm{BF}_{4}$ giving the diiodo compounds 22 and 23, respectively (Scheme 6) [13]. The evolution of this transformation was monitored by ${ }^{1} \mathrm{H}$ and ${ }^{13} \mathrm{C}$ NMR spectroscopy. On the basis of the information provided by these experiments, an intermediate iodolium ion of the type represented by $\mathbf{2 4}$ was 
characterized, providing support for the remarkable regio-and stereocontrol observed in the reaction. Also, of synthetic interest, is the fact that enyne $\mathbf{1 9}$ can be used to easily elaborate enediyne fragments. Thus, 25 was synthesized in three steps from phenylacetylene, comprising conversion of $\mathrm{C}(\mathrm{sp})-\mathrm{H}$ into $\mathrm{C}\left(\mathrm{sp}\right.$ )-SiMe $\mathrm{Bu}_{2}^{\mathrm{t}}$, dimerization mediated by $\mathrm{IPy}_{2} \mathrm{BF}_{4}$ (Scheme 5), and palladium catalyzed coupling with TMSA (trimethylsilylacetylene) (Scheme 6)

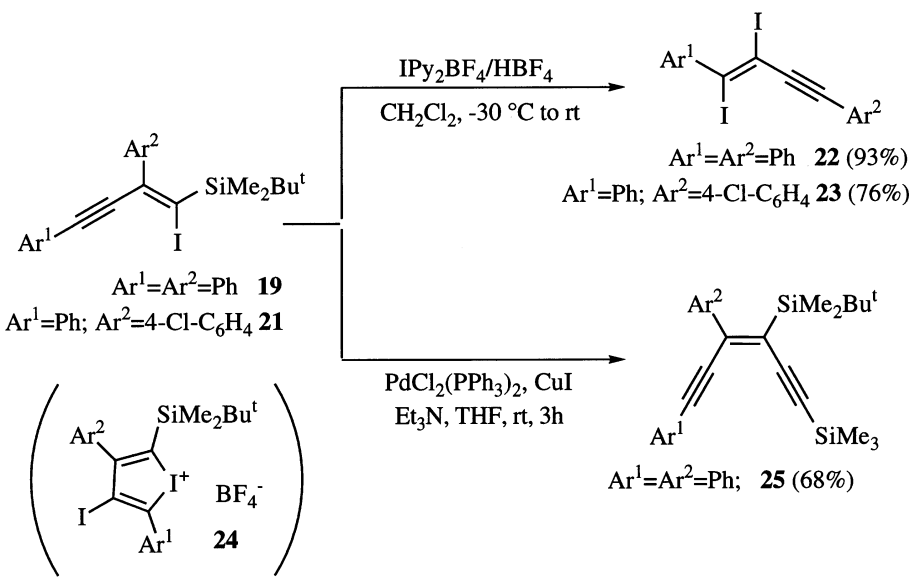

\section{Scheme 6}

The use of phenylsulfide as a masking functionality for the $\mathrm{C}(\mathrm{sp})-\mathrm{H}$ bond has resulted in the discovery of a new and valuable synthetic sequence. Using this activating group, alkynes with aliphatic side chains, as the case of $\alpha, \omega$-diynes, react efficiently with $\mathrm{IPy}_{2} \mathrm{BF}_{4}$, a process that fails using the related iodo-and silyl substituted precursors. The conversion of $\mathbf{1 4}$ into 20 (Scheme 5) upon treatment with $\mathrm{IPy}_{2} \mathrm{BF}_{4}$ happens very rapidly at $-80^{\circ} \mathrm{C}$, again only one equivalent of acid is used in this case to activate the reagent [14]. The cyclization mode corresponds to an exo-endo approach of the alkyne components [15], an unusual process (most transition-metal mediated cyclizations take place according to an exo-exo alkyne coupling) [16] that has been almost exclusively reported when the alkyne components are constrained inside macrocyclic structures [17], or in polymerization processes prompted by metathesis catalysts [18]. The formation of the reaction product involves two transformations, first an alkyne-alkyne coupling occurs then, the intermediate ion is trapped by the aryl ring of the phenylsulfide group to give the tricyclic structure, in a Friedel-Crafts-like ring closure, defining a new domino sequence [19].

As a common feature, the coupling reactions depicted in Scheme 5 incorporate the iodine atom that promotes or catalyzes the transformation into the assembled skeleton, thus making possible its eventual use as a building-block $[13,14]$ and, in that sense, establishes a net difference of this approach with most other synthetic approaches to these targets, mainly organometallic, in which an hydrogen rather than an iodine is placed in the structure of the final reaction product.

The unique iodinating capability of the reagent towards aromatic systems represents another significant characteristic of its reactivity. In fact, using $\mathrm{IPy}_{2} \mathrm{BF}_{4}$ it is possible to accomplish, at room temperature, the monoiodination of a wide set of representative arenes, as illustrated Scheme 7 [20-22].

The reaction with several model compounds [20] includes successful examples of iodination of very reactive molecules, such as the acid-labile indole nucleus $\mathbf{2 8}$, and aromatic compounds with electron releasing substituents, such as anisole (see conversion of $\mathbf{2 6}$ into 32). Under virtually the same conditions, arenes substituted by electron withdrawing groups (see conversion of 30 and $\mathbf{3 1}$ into $\mathbf{3 6}$ and 37, respectively) can be efficiently iodinated, by simply extending the reaction time. Also of interest, is the iodination of substituted anilines on a multigram scale, that has allowed us to establish a new protocol to synthesize 5-and 5,7-disubstituted indoles from commercially available anilines [21]. The iodination has been also applied to label aromatic amino acids (see conversion of 29 into 35, Scheme 7) and bioactive peptides, either in solution [22] or in the solid phase [23].

In summary, the $\mathrm{IPy}_{2} \mathrm{BF}_{4}$ reagent combines great synthetic potential to solve basic organic transformations with the capability to delineate novel coupling reactions with great selectivity and, 


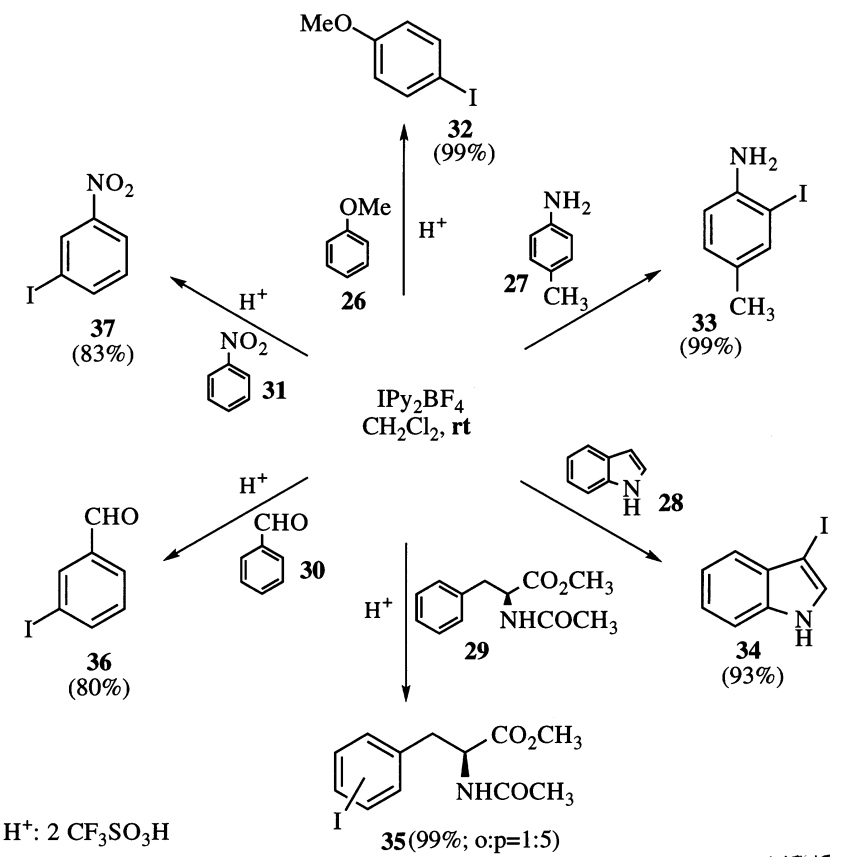

\section{Scheme 7}

though with the restrictions imposed by the substitution pattern, opening alternatives to the usual transition metal based transformations.

\section{ACKNOWLEDGEMENTS}

I wish to express my sincere appreciation to my collaborators, whose names appear in the references. Financial support from DGES (PB97-1271) is gratefully acknowledged.

\section{REFERENCES}

1 R. H. Seevers, R. E. Counsell. Chem. Rev. 82, 575 (1982).

2 For recent reviews see for instance: (a) P. J. Stang, V. V. Zhdankin. Chem. Rev. 96, 1123 (1996). (b) T. Umemoto. Chem. Rev. 96, 1757 (1996). (c) A. Varvoglis. Tetrahedron 53, 1179 (1997). (d) A. Varvoglis, S. Spyroudis. Synlett, 221 (1998).

3 J. Barluenga, J. M. González, P. C. Campos, G. Asensio. Angew. Chem. Int. Ed. Engl. 24, 319 (1985).

4 J. Barluenga, P. C. Campos, J. M. González, J. L. Suárez, G. Asensio. J. Org. Chem. 56, 2234 (1991).

5 J. Barluenga, L. J. Alvarez-Garcia, J. M. González. Tetrahedron Lett. 36, 2153 (1995).

6 J. Barluenga, L. J. Alvarez-Garcia, G. P. Romanelli, J. M. González. Tetrahedron Lett. 38, 6763 (1997).

7 (a) J. Barluenga, M. A. Rodríguez, J. M. González, P. J. Campos. Tetrahedron Lett. 27, 3303 (1986). (b) J. Barluenga, M. A. Rodríguez, P. J. Campos. J. Org. Chem. 55, 3104 (1990).

8 (a) J. Barluenga, M. A. Rodríguez, P. J. Campos, G. Asensio. J. Am. Chem. Soc., 110, 5567 (1988). (b) J. Barluenga, P. J. Campos, F. López, I. Llorente, M. A. Rodríguez. Tetrahedron Lett. 31, 7375 (1990).

9 J. Barluenga, J. M. González, P. C. Campos, G. Asensio. Angew. Chem. Int. Ed. Engl. 27, 1546 (1988).

10 (a) J. Barluenga, M. A. Rodríguez, J. M. González, P. J. Campos. Tetrahedron Lett. 31, 4207 (1990). (b) M. B. Goldfinger, T. M. Swager, J. Am. Chem. Soc. 116, 7895 (1994).

11 J. Barluenga, J. M. González, I. Llorente, P. J. Campos, Angew. Chem. Int. Ed. Engl. 32, 893 (1993).

12 B. M. Trost. Angew. Chem. Int. Ed. Engl. 34, 259 (1995).

13 J. Barluenga, I. Llorente, L. J. Alvarez-García, J. M. González, P. J. Campos, M. R. Díaz, S. García-Granda. J. Am. Chem. Soc. 119, 6933 (1997). 
14 J. Barluenga, G. P. Romanelli, L. J. Alvarez-García, I. Llorente, J. M. González, E. García-Rodríguez, S. GarcíaGranda. Angew. Chem. Int. Ed. Engl. 37, 3136 (1998).

15 K. Tamao, S. Yamaguchi, M. Shiro, J. Am. Chem. Soc. 116, 11715 (1994).

16 I. Ojima, M. Tzamarioudaki, Z. Li, R. J. Donovan. Chem. Rev. 96, 635 (1996).

17 (a) Q. Zhou, P. J. Carroll, T. M. Swager. J. Org. Chem. 59, 1294 (1994). (b) X. Gu, M. B. Sponsler. Tetrahedron Lett. 37, 1571 (1996). (c) H. Weigl, R. Gleiter. Tetrahedron Lett. 38, 1541 (1997).

18 M. Schuster, S. Blechert. Angew. Chem. Int. Ed. Engl. 36, 2037 (1997).

19 L. F. Tietze. Chem. Rev. 96, 115 (1996).

20 (a) J. Barluenga, J. M. González, M. A. García-Martín, P. J. Campos, G. Asensio. J. Chem. Soc., Chem. Commun. 1016 (1992). (b) J. Barluenga, J. M. González, M. A. García-Martín, P. J. Campos, G. Asensio. J. Org. Chem. 58, 2058 (1993).

21 J. Ezquerra, C. Pedregal, C. Lamas, J. Barluenga, M. Pérez, M. A. García-Martín, J. M. González. J. Org. Chem. 61, 5804 (1996).

22 J. Barluenga, M. A. García-Martín, J. M. González, P. Clapés, G. Valencia. Chem. Commun. 1505 (1996).

23 G. Arsequell, G. Espuña, G. Valencia, J. Barluenga, R. Pérez Carlón, J. M. González. Tetrahedron Lett. 39, 7393 (1998). 